\title{
Transcatheter aortic valve replacement and Candida parapsilosis endocarditis: a deadly combination
}

\author{
Susana Ortega-Silva ${ }^{1}$, Willian A. Ortiz-Solis ${ }^{1}$, Diego Neach de la Vega ${ }^{1}$, Nilda Espinola-Zavaleta ${ }^{2,3 *}$, America G. Urbina-Vazquez ${ }^{1}$, Antonio \\ R. Bailon-Sotelo ${ }^{1}$ and Abraham Villalobos-Flores ${ }^{1}$
}

${ }^{1}$ Department of Cardiology, National Institute of Cardiology Ignacio Chavez, Mexico City, Mexico

${ }^{2}$ Department of Nuclear Cardiology, National Institute of Cardiology Ignacio Chavez, Mexico City, Mexico

${ }^{3}$ Department of Echocardiography, ABC Medical Center, I.A.P., Mexico City, Mexico

\begin{abstract}
Introduction: Transcatheter aortic valve infective endocarditis is a life-threatening complication. Transcatheter aortic valve infective endocarditis (TAVIE) mortality caused by Candida parapsilosis is unknown.

Clinical Case: We present a case of a 73-year-old female diagnosed with severe aortic stenosis secondary to bicuspid aortic valve and coronary artery disease with a significant lesion of the proximal left anterior descending artery, treated with one sirolimus-eluting stent and transcatheter aortic valve replacement. A month later she began with persistent fever and hypotension. A transesophageal echocardiogram (TEE) showed vegetations adhered to the transcatheter aortic valve prosthesis with a moderate posterior paravalvular leak. Positive blood cultures were obtained with Candida parapsilosis isolation. She was treated with Amphotericin B plus voriconazole and underwent surgical aortic valve replacement. Unfortunately, surgery was complicated with aortic and coronary arteries dissection, cardiorespiratory arrest and death.
\end{abstract}

The transesophageal echocardiography had an excellent correlation with surgical findings.

\section{Introduction}

Transcatheter aortic valve infective endocarditis (TAVIE) is an uncommon but life-threatening complication, with an incidence around $1 \%$ per patient-year. Fungal endocarditis accounts only 2 to $5 \%$ of cases [1]. Candida parapsilosis is the second most common fungus and many internal and external factors are related to the infection as contaminated external devices or fluids, the organism growth capabilities, immunocompromised individual state, and with skin, respiratory and gastrointestinal infections [2]. This fungus has a peculiar pathogenetic mechanism involving biofilm formation on indwelling devices, including prosthetic valves [3]. Transesophageal echocardiography is the study of choice in prosthetic valve endocarditis when the transthoracic echocardiogram is inconclusive with a sensitivity and specificity close to $100 \%$ ( $92 \%$ and $90 \%$, respectively) [4].

\section{Case presentation}

A 73-year-old female with a history of systemic hypertension, thyroidectomy for multinodular goiter and breast cancer in remission for 10 years. She began with progressive dyspnea and angina. She was diagnosed with severe aortic stenosis secondary to bicuspid aortic valve and coronary artery disease with a significant obstructive lesion of the proximal left anterior descending artery. She was treated with one sirolimus-eluting stent and transcatheter aortic valve replacement (TAVR) with an Evolut ${ }^{\mathrm{mi}} \mathrm{R}$ valve. During her hospitalization she developed acute appendicitis that was managed nonoperatively with adequate response and no other complications were detected. A month later she began with fever up to $39^{\circ} \mathrm{C}$ with an Escherichia coli positive urine culture isolation, she received antibiotic treatment without improvement. Few days later she presented to the emergency department with persistent fever and hypotension. Severe pancytopenia was identified and positive blood cultures were obtained with Candida parapsilosis isolation. A transesophageal echocardiogram (TEE) was performed which identified the presence of vegetations adhered to the transcatheter aortic valve prosthesis (TAVI) with a moderate posterior paravalvular leak (Clip 1 and 2). A bone marrow aspiration and culture were performed which reported the presence of hyphae and pseudohyphae with confirmation of Candida parapsilosis infection (Figure 1). Amphotericin B and filgrastim were given without improvement so she was transferred to our institution. The physical examination showed blood pressure of $100 / 60 \mathrm{mmHg}$, pulse $83 \mathrm{bpm}$, $\mathrm{SaO} 291 \%$ and temperature $37^{\circ} \mathrm{C}$. Cardiovascular exam was notable due to a palpable thrill in the chest, a grade IV diastolic murmur and diffuse pulmonary rales in both lungs. Laboratory findings showed hemoglobin $7.8 \mathrm{~g} / \mathrm{dl}(12.7-16.3 \mathrm{~g} / \mathrm{dl})$, leukocytes $3.4 \times 10^{3}\left(3.5-10.3 \times 10^{3}\right)$ neutrophil $2 \times 10^{3}\left(1.7-6.5 \times 10^{3}\right)$, platelets $57 \times 10^{3}\left(150-500 \times 10^{3}\right)$, creatinine $0.63 \mathrm{mg} /$ $\mathrm{dl}(0.6-1.2 \mathrm{mg} / \mathrm{dl})$, potassium $2.5 \mathrm{mEq} / \mathrm{l}$ (3.5-5.1 mmol/l), NT-proBNP $4873 \mathrm{pg} / \mathrm{ml}(15-125 \mathrm{pg} / \mathrm{ml})$ and C-reactive protein (CRP) $40 \mathrm{mg} / \mathrm{L}$ (1-

${ }^{\star}$ Correspondence to: Nilda Espinola-Zavaleta, $\mathrm{MD}, \mathrm{PhD}$, Nuclear Cardiology Department, National Institute of Cardiology Ignacio Chavez, Juan Badiano No 1, Colonia Seccion XVI, Tlalpan, P.C. 14080, Mexico City, Mexico, Tel: +525555732911; E-mail: niesza2001@hotmail.com

Key words: candida parapsilosi, infective endocarditis, transcatheter aortic valve prosthesis, transesophageal echocardiography

Received: August 28, 2019; Accepted: September 23, 2019; Published: September 26, 2019 


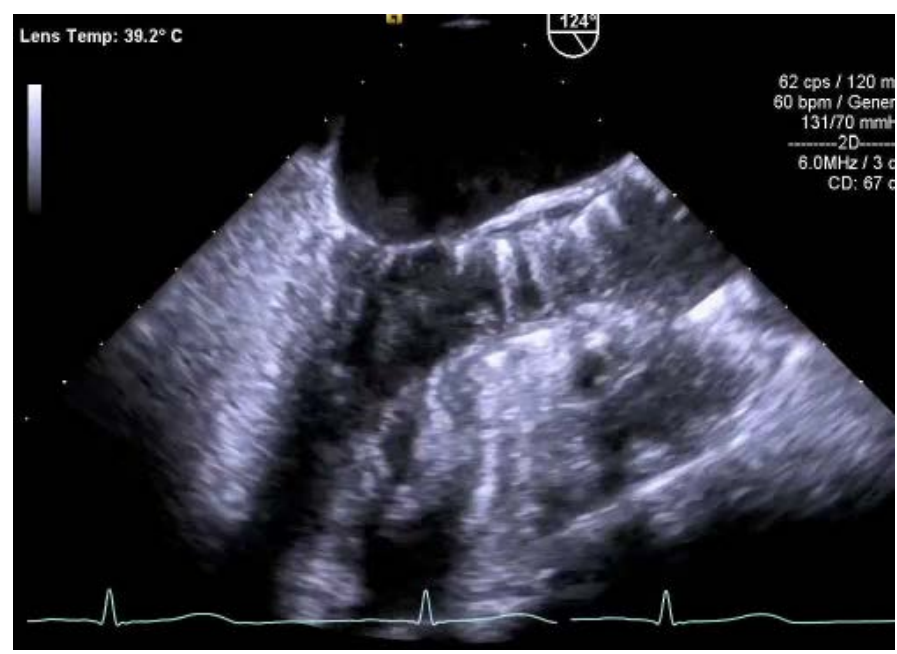

Clip 1. Two-dimensional transesophageal echocardiogram at $124^{\circ}$, showing vegetations in the transcatheter aortic valve prosthesis

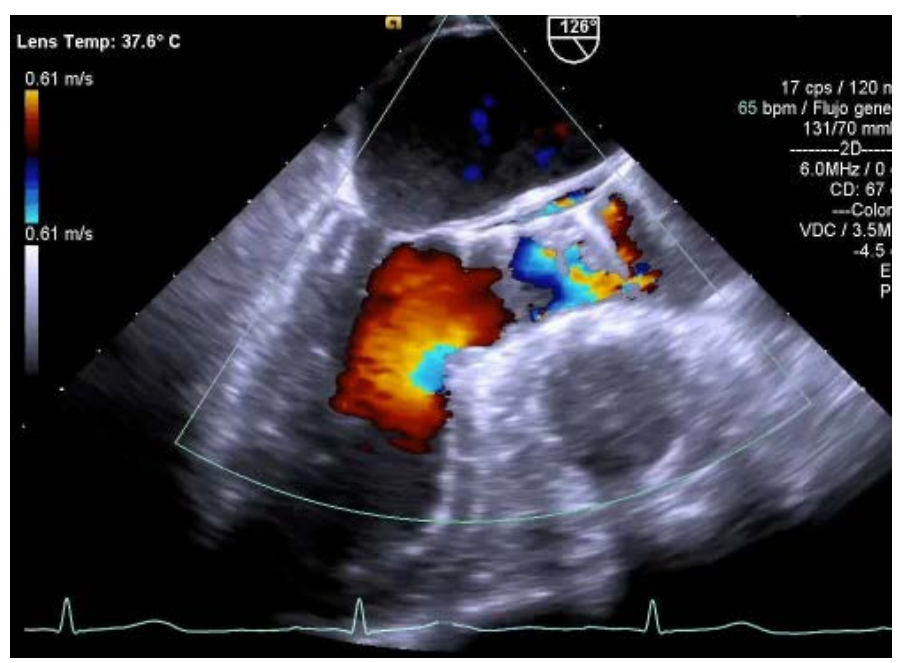

Clip 2. Color-flow transesophageal echocardiogram at $126^{\circ}$, with moderate posterior paravalvular leak

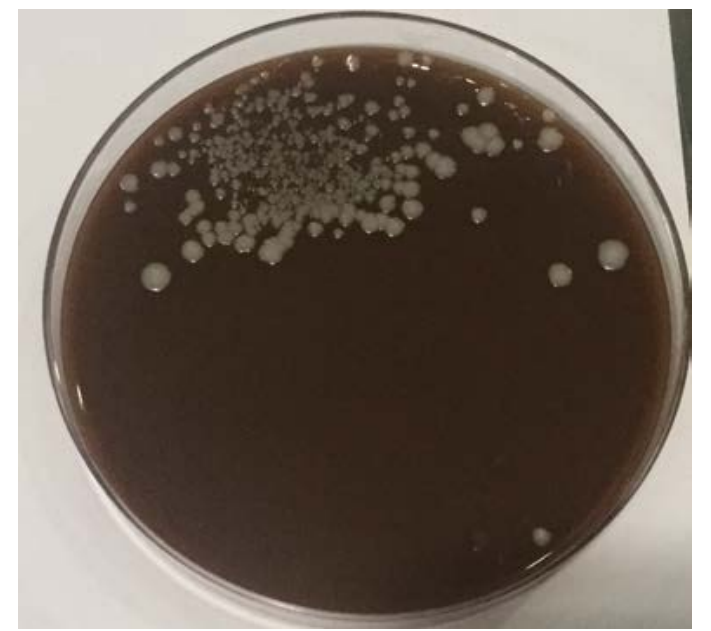

Figure 1. In the CHROM agar Candida ${ }^{\circledR}$, medium, specimens of Candida parapsilosis were identified, which are macroscopically characterized by being creamy from white to pink, round or oval from 3 to 7 microns in diameter
$3 \mathrm{mg} / \mathrm{L})$. She continued antifungal intravenous therapy and underwent surgical aortic valve replacement. A good correlation between TEE and surgery was found (Figures 2 and 3 ). The procedure was complicated with aortic and coronary arteries dissection at the time the prosthetic valve was removed. Bentall and De Bono surgery and coronary artery bypass procedures were performed without success, which lead to cardiorespiratory arrest and death.

\section{Discussion}

Infective endocarditis (IE) is a high mortality disease and remains associated with severe complications and high in-hospital mortality rate despite an appropriate antibiotic therapy, surgical intervention or other recently improvements in its management. It is reported a 50 times higher IE risk in patients with a prosthetic valve compared with the general population $[5,6]$. The presence of prosthetic valves are the most important risk factors for developing fungal IE, but abdominal surgery or abdominal infection has also been described as a risk factor $[7,8]$, which was relevant in our patient. We documented the isolation of Candida parapsilosis in TAVI, in bone marrow and in blood cultures. To our knowledge this is the third case of Candida parapsilosis in TAVI reported in the literature (Table 1).

TAVIE is an uncommon but life-threatening complication. Nowadays is becoming more prevalent with an incidence around $1 \%$ per patient-year, however up to $3.1 \%$ has been detected in small studies [7]. Amat-Santos IJ, et al. documented that respiratory or skin infections, dental, urological or gastrointestinal interventions were the main sources of endocarditis but remained undetermined in as many
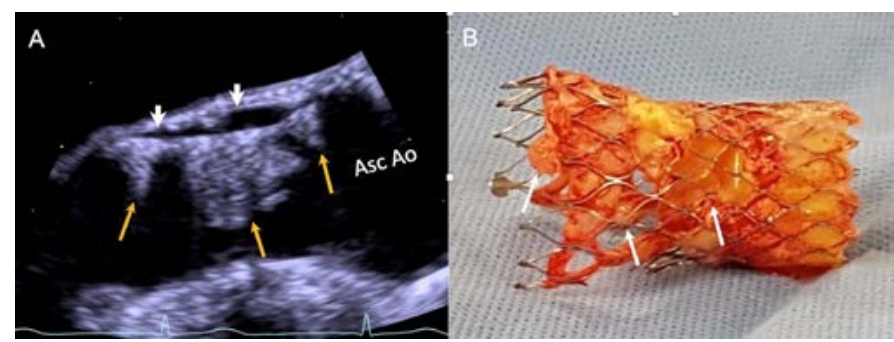

Figure 2. A- Transesophageal two-dimensional zoom of the transcatheter aortic valve prosthesis with multiple vegetations (orange arrows) and paravalvular leak (white arrowheads). B- Transcatheter aortic valve prosthesis with multiple vegetations (orange arrows). Asc $\mathrm{Ao}=$ Ascending aorta

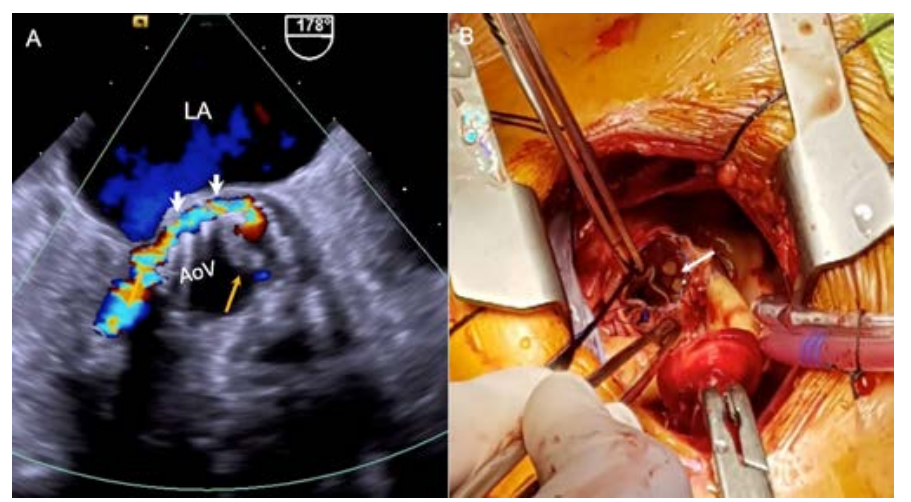

Figure 3. A- Two-dimensional and color flow-transesophageal image of transcatheter aortic valve prosthesis with vegetations (orange arrows) and moderate posterior paravalvular leak (white arrowheads). B-Surgical view of the transcatheter aortic valve prosthesis with vegetations (orange arrow). LA: left atrium 
Table 1. Reported cases of TAVR-EI by Candida parapsilosis

\begin{tabular}{|c|c|c|c|c|c|c|}
\hline Reference & Data & Age/ Gender & Valve & $\begin{array}{c}\text { Duration since } \\
\text { TAVR }\end{array}$ & Antifungal therapy & Surgery \\
\hline$[3]$ & 2018 & $80 / \mathrm{M}$ & SAPIEN $23 \mathrm{~mm}$ & 4 months & $\begin{array}{c}\text { L-AMB, MCFG + } \\
\text { FLCZ }\end{array}$ & Yes \\
\hline$[9]$ & 2019 & $76 / \mathrm{M}$ & ND & 9 months & Yes \\
\hline Our case & 2019 & $73 / \mathrm{F}$ & $\begin{array}{c}\text { CoreValve Evolut R } \\
26 \mathrm{~mm}\end{array}$ & 30 days & L-AMB + VCZ & Yes \\
\hline
\end{tabular}

Abbreviations: TAVR: transcatheter aortic valve replacement; IE: infective endocarditis; M: male; F: female; L-AMB: liposomal amphotericin B; VCZ: voriconazol; MCFG: micafungin, FLCZ: fluconazole; ND: non-determined

as $50 \%$ of the patients [6]. Regueiro A, et al. [9] found that the male gender, diabetes mellitus and moderate to severe aortic regurgitation are the risks factors. In this study, patients who developed TAVIE had rates of in-hospital and two-year mortality of $36 \%$ and $66.7 \%$, respectively. The median time of developing symptoms was around 5 months and enterococci species and staphylococcus aureus were the most frequently isolated microorganisms.

IE secondary to fungal microorganisms is a high mortality rate disease and the vast majority of cases are caused by Candida spp. and Aspergillus spp. Several case reports documented a fungal TAVIE incidence of $0.8 \%$ up to $1.9 \%$ with a mortality rate of $50 \%$. Very few were caused by Candida parapsilosis infection $[3,10]$, which was the etiology in our case This microorganism is found in nails and skin as a part of normal microbial flora but can cause invasive infection without preceding colonization in cardiac surgery or transvenous procedures by biofilms on implanted devices [3]. Actually, valve replacement and antifungal therapy is the treatment of choice in IE but there is not yet formal recommendation of TAVIE $[4,5]$.

Actually, with the large number of indications for TAVR, we are sure that soon we will have specific recommendations to deal successfully with this type of cases.

\section{Highlights}

1. Transcatheter aortic valve infective endocarditis is a life-threatening complication

2. The transesophageal echocardiography has an excellent correlation with surgical findings in the diagnosis of transcatheter aortic valve infective endocarditis.

\section{Funding}

No Fundings

\section{Acknowledgement}

No Acknowledgement

\section{References}

1. Madakshira M, Bal A, ShivaPrakash (Candida parapsilosis endocarditis in an intravenous drug abuser: an autopsy report. Cadiovasc Pathol 36: 30-34

2. Trofa D1, Gácser A, Nosanchuk JD (2008) Candida parapsilosis, an emerging fungal pathogen. Clin Microbiol Rev 21: 606-625. [Crossref]

3. Morioka H, Tokuda Y, Oshima H (2019) Fungal endocarditis after transcatheter aortic valve replacement (TAVR): Case report and review of literature. $J$ Infect Chemother 25: 215-217.

4. Gilbert H, Patrizio L, Antunes M (2015) ESC Scientific Document Group, 2015 ESC Guidelines for the management of infective endocarditis: The Task Force for the Management of Infective Endocarditis of the European Society of Cardiology (ESC) Endorsed by: European Association for Cardio-Thoracic Surgery (EACTS), the European Association of Nuclear Medicine (EANM). Eur Heart J 36: 3075-3128.

5. Nishimura RA, Otto CM, Bonow R (2017) AHA/ACC Focused Update of the 2014 AHA/ACC Guideline for the Management of Patients With Valvular Heart Disease. JACC 70: 252-289.

6. Amat-Santos IJ, Ribeiro HB, Urena M (2015) Prosthetic valve endocarditis after transcatheter valve replacement: A systematic review. JACC Cardiovasc Interv 8: 334 346 .

7. Van Asbeck EC, Clemons KV, Stevens DA (2009) Candida parapsilosis: a review of its epidemiology, pathogenesis, clinical aspects, typing and antimicrobial susceptibility. Crit Rev Microbiol 35: 283-309.

8. Antinori S, Milazzo L, Sollima S, Galli M, Corbellino M (2016) Candidemia and invasive candidiasis in adults: A narrative review. Eur J Intern Med 34: 21-28. [Crossref]

9. Regueiro A, Linke A, Latib A (2016) Association between transcatheter aortic valve replacement and subsequent infective endocarditis and in-hospital death. $J \mathrm{Am} \mathrm{Med}$ Assoc 316: 1083-1092.

10. Carrel T, Eberle B (2019) Candida Endocarditis after TAVR. $N$ Engl J Med 380: e1 [Crossref]

Copyright: (C2019 Ortega-Silva S. This is an open-access article distributed under the terms of the Creative Commons Attribution License, which permits unrestricted use, distribution, and reproduction in any medium, provided the original author and source are credited. 